\title{
Bow \& Tea-An Unleashed Device Using Everyday Components and Technology
}

\author{
Eunjoo Shin \\ Interactive Design, College of Fine Art and Design, Incheon Catholic University \\ 12 Haesongro, Yeonsu-Gu, Incheon 406-840, Korea \\ ejoo@iccu.ac.kr
}

\begin{abstract}
Device Art, initiated in 2004 by Machiko Kusahara, is a new form of media art in Japan, which integrates art and technology, as well as design, entertainment, and popular culture. Each device is specially invented for artistic purposes, and it can be considered as an artwork on its own as it becomes the content itself. This paper explores the idea of the unleashed device - artists using everyday components and objects, and mixing them together with media technologies; then these artists apply their unique imaginations, uniting art, design, and technology. To identify the underlying potential of unleashed devices in a context that spurs the artistic sensibility to participate in the interplay between varied interactive devices, and also to be fruitful in terms of ludic, playful technology and performative experiences, I will review some related works and discuss Bow \& Tea as a case study.
\end{abstract}

Keywords: Unleashed Device, Mechatronic Device, Entertainment Experience, Interactivity, Daily Object

\section{Introduction}

Technology is having a big impact on human beings, including in enabling progress and the creation of new experiences. The ongoing integration of new devices with technologies has had a profound effect on the relationship between people and products [1]. In general, we have seen a lot of devices that have been mostly general-purpose items. However, things got really exciting when hardware and the software came together and we began to really make products. Moreover, rapidly evolving technologies have enabled opportunities to create new devices to experience art. Things became more personal as they become more product like, opening up a whole new range of possible products for us to design. Then, behaviors really came into it. Now we are talking about special-purpose products where we can design the controls.

Initiated in 2004 by Machiko Kusahara, "Device Art" is a new form of media art in Japan that integrates art and technology, as well as design, entertainment, and popular culture in the form of mechatronic devices. Each device has been specially invented for artistic purposes. As a result, these artists have developed unique creations in the genre of Device Art. Their work can be considered artwork on its own as it becomes the content itself. Using media technology, Device Art brings a sense of playfulness or wonder with it. Thus, this makes it possible for Design Art to be shown or commercialized outside of museums and galleries, even if it involves a serious theme [2]-3].

This paper explores the idea of unleashed devices - artists using everyday components and objects, and mixing them together with media technologies, and, in applying their unique imaginations, uniting art, design, and technology. In this research, I move beyond a dictionary of the definition of devices: "A thing made or adapted for a particular purpose, especially a piece of mechanical or electronic equipment" [10]. Rather, I want to study how designed devices are tailor made and constructed to provoke participation and 
engage artists in different ways.

I want to identify the underlying potential of unleashed devices in a context that spurs the artistic sensibility to participate in the interplay between varied interactive devices. I also want to be fruitful in terms of ludic, playful technology and performative experiences that occur every day. Therefore, I will review the related works and discuss Bow \& Tea, which is being exhibited in a "Chi-Tek" showcase at the Victoria \& Albert Museum and Watermans Art Centre, as a case study.

\section{Related Works}

Powerful, technology-driven electronics are being used widely. Their user-friendly interface designs have enabled people to easily use them. As a result, people are able to enjoy new technologies, and scientific knowledge is not required any more. For instance, GPS has become essential for drivers, and a various apps have become a convenient means of getting information and communication. These apps involve brilliant ideas, which make users enthusiastic about using them and enjoying their content, and then people have new experiences. The Nintendo Wii gaming technology allows users' physical reactions to be directly reflected in a 3D virtual reality game. In daily life, we use highly developed electronics as personal, handy devices.

Now artists also use media technology to realize their ideas for creative, entertaining purposes, and the electronic devices they create are sold commercially (e.g., Maywa Denki, Toshio Iwai). A platform for producing software and hardware for artists and designers has been developed and is widely offered as an open source platform under the creative common licenses. This movement brings about the possibility of artists and designers fulfilling their imagination by manipulating diverse existing objects and products.

\subsection{Tenori-On}

Designed by Toshio Iwai (a media artist), Tenori-On was conceived as an innovative, digital instrument. Tenori-On is constructed of rounded, polished magnesium. It consists of an intuitive, visual interface that is for playing a MIDI controller, a tone generator, and a sampler. Users create a pattern on a $16 \times 16$ LED matrix of opaque plastic buttons, which play music that result in light graffiti. With the six performance and sound/light modes, users can compose new music by editing and saving the sound sources, which are produced by light patterns stored in the memory as the 16 blocks of programing data [11].

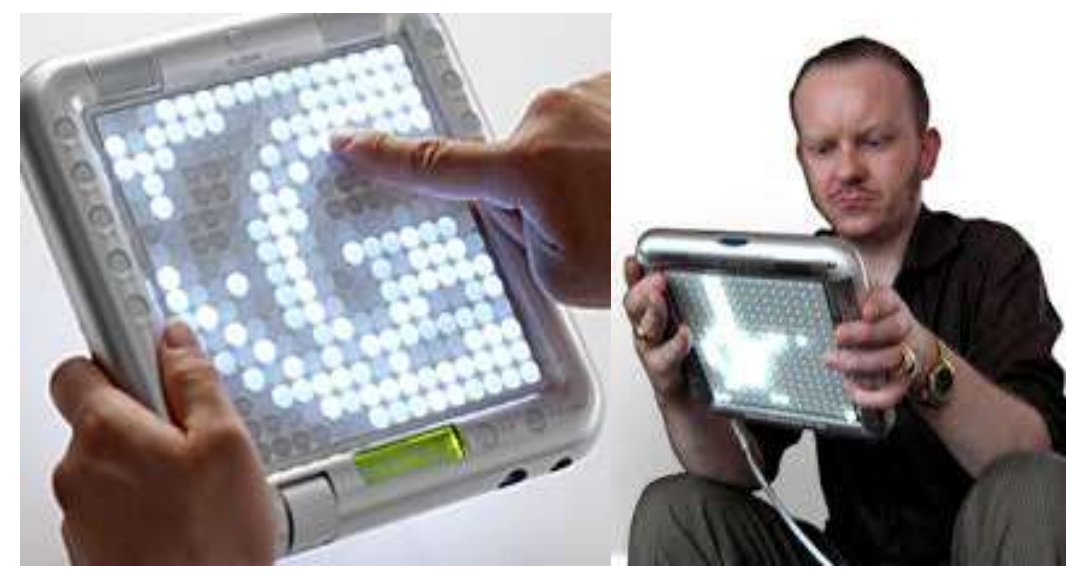

Figure 1. Tenori-On

Tenori-On is as much about visuals as it is about sound, and its graphical lights are presented to both the performer and audience. Thus, it indicates the entertaining 
characteristic of editing and composing music, light patterns, and performance with a specially designed interface that is easy to operate. It resembles an object from the Starship Enterprise; it is an unprecedented, hi-tech cyber toy, which generates a synthesis of sounds and drawings. Although MIDI devices have been used for making sounds, a new musical MIDI device has been invented by integrating the idea of drawing lights onto existing MIDI devices using technology. This brilliant device broadens our experience in a unique way.

\subsection{Bitman}

Bitman is one of Maywa Denki's art products. Produced in collaboration with Royota Kuwakubo, Bitman is a small device that users can wear. It features a $7 \mathrm{~cm} \times 7 \mathrm{~cm}$ white plastic frame with an $8 \times 8$ matrix of red LEDs. It displays an image made up of pixels of a figure who is recognizable from early computer games. The device is hung with a chain around one's neck. The bitmap image shows a real-time animation of Bitman, trying to keep his balance in the revolving world. By shaking the unit, a small pixeled man jumps around, changes his orientation, and produces some accompanying buzzing noises.

The movement of the digital bitmap character is created in real time in response to an acceleration signal, and by tilting the unit with built-in, embedded sensors. It can also be used as a watch or alarm, and users can enter and display messages. This simple, functional toy has become a very popular item commercially [12]. Bitman is based on the functioning of a watch or alarm, but the real time character's moves interacting with the user's bodily movement reminds us of a retro game through an entertaining experience.
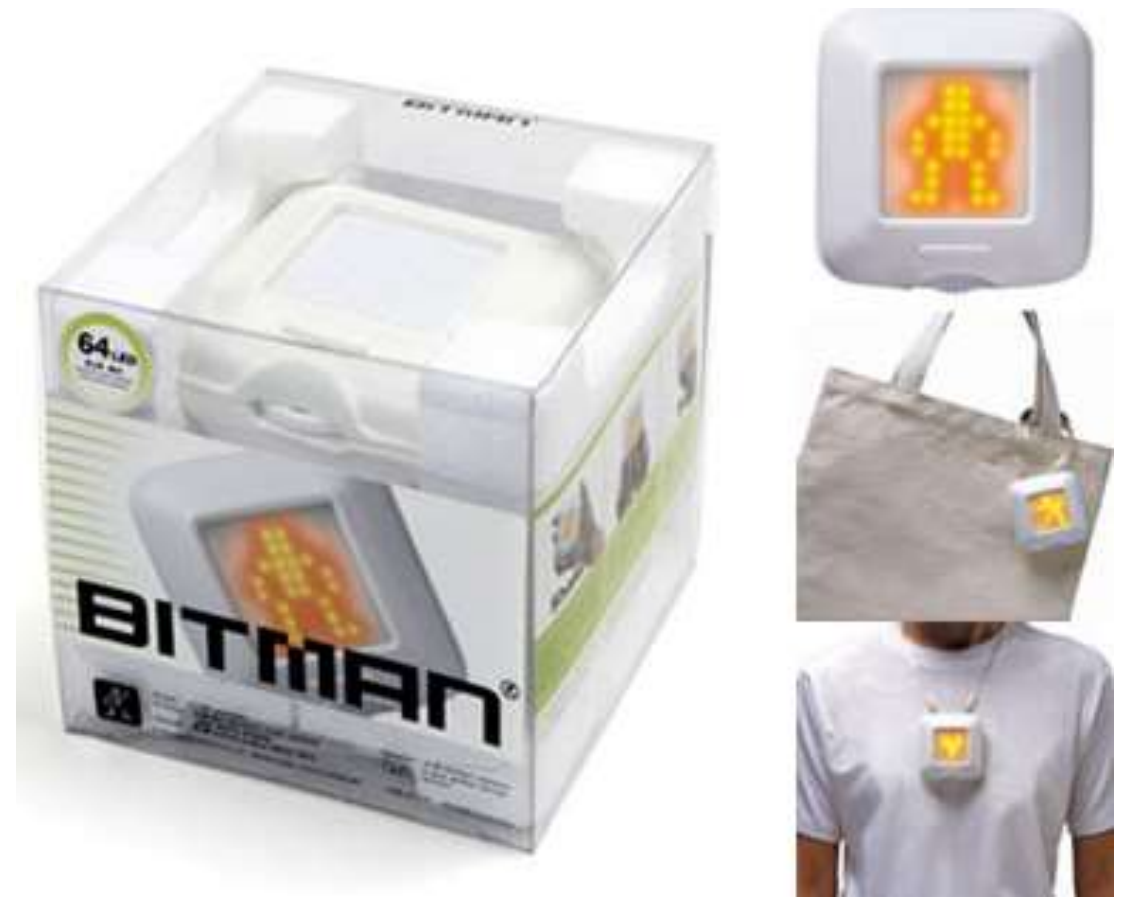

Figure 2. Bitman 


\subsection{Otamatone}

The Otamatone is a toy-like musical instrument, which was developed by Maywa Denki and Cube Works, a toy company. It consists of two parts: a long, thin tail and a rounded head resembling a tadpole. The long tail acts like a keyboard, which emits different pitches, and the tadpole's head changes the tone by opening and closing its mouth. To play it, one hand squeezes the tadpole's head, and the other hand controls the pitch of the tune by pressing the stem-switch on a tail controller. Varying the pressure on the head creates a wah-wah sound effect, and shaking the neck by slightly changing pressure on the tadpole's head creates a vibrato effect [13].

An Otamatone is kind of synthesizer, but it has also been compared to a theremin because of its weirdness. This peculiar instrument has many fans all over the world; it is easy to learn to play, and it has an analog of digital sound motion. It does not function perfectly in terms of playing exact sounds, as would be required for symphony orchestras, but it is a whimsical piece of fun and playfulness for users. Unlike the previously mentioned Tenori-On, Otamatone is focused on a humorous experience that evokes the look of a tadpole opening its mouth to sing.
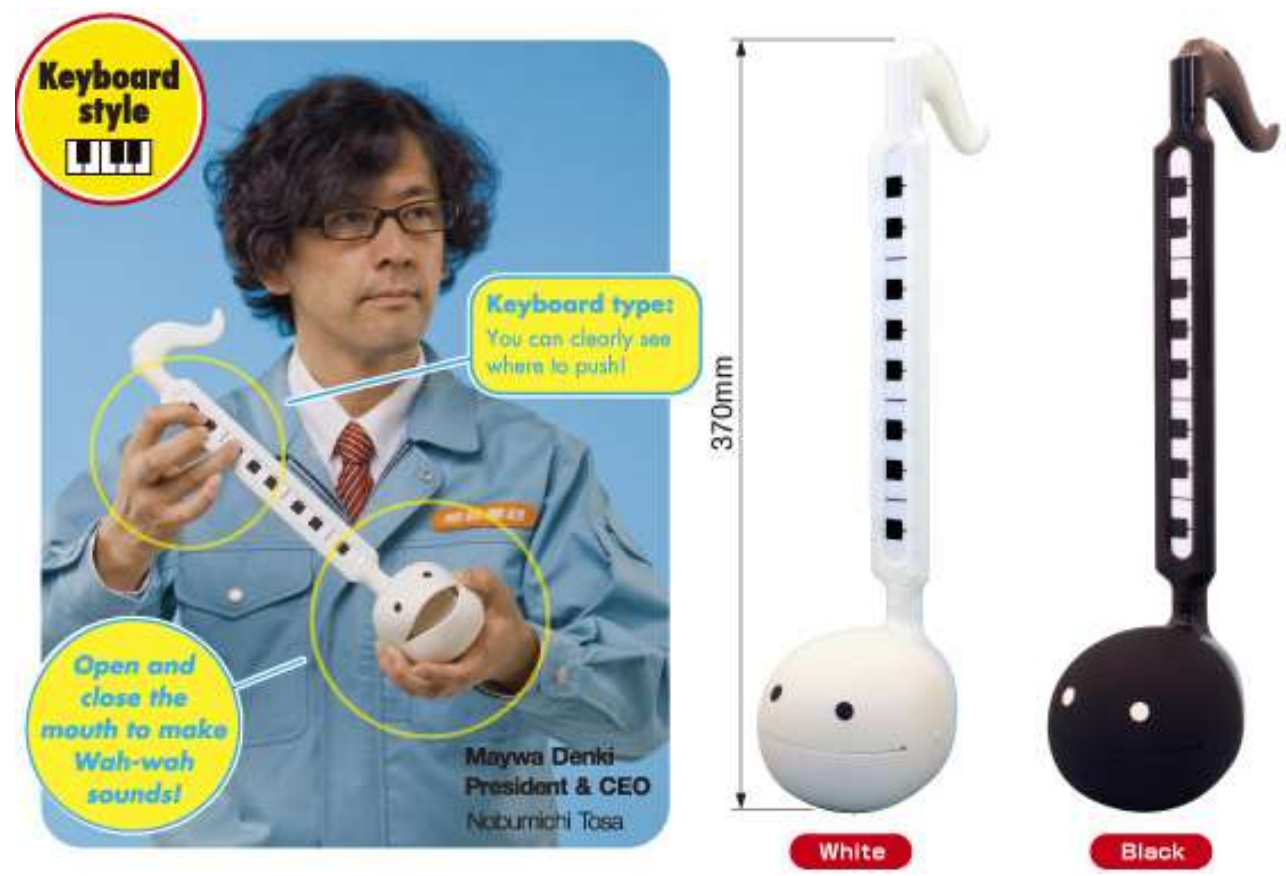

Figure 3. Otamatone

\section{Bow \& Tea}

\subsection{Background}

Using both the latest technology and everyday objects, newly invented devices allow users to enjoy their mesmerizing experiences through physical interactivity. These changes highlight how important it is to provide narrative experiences through the interactivity between users and devices [6]. And the role of users and their participation is emphasized. As a result, devices can allow a user to be the protagonist and co-producer of a narrative experience instead of a passive user of a device's functionality; this gives the device meaning [1].

Furthermore, the boundaries of art, design, and technology have also blurred as the former two integrate with technology. It is important that Bow \& Tea can be appreciated 
as art as well as a product in terms of an unleashed device in which artists release their unique imaginations, uniting art, design, and technology. In this respect, Bow \& Tea is focused on humorous experiences through an interface designed to make and serve tea.

\subsection{Configuration}

Bow \& Tea, an electronic device, is based on a tea ceremony, which is a cultural activity that involves the ceremonial preparation and presentation of tea in Asian culture. At a very basic level, tea ceremonies are a formalized process, which has been refined to yield the best taste. A whole set of rituals, tools, and gestures are used during tea ceremonies [14].

Bow \& Tea allows users to make tea for not only themselves but also others by controlling the speed of the dropping tea leaves as well as their amount in a teacup. When users move the tea plate in the center up and down, it performs like a bow for someone. As a kind of a witty metaphor, it encourages the server to give thanks to the tea maker in a polite manner. Bow \& Tea lets users produce their own ritualistic stories with an interface that suggests unusual tea making. And the entertainment process is like a playing a humorous game. Through manipulating an existing tea-making machine with the unique use of technology, users can share an enjoyable tea break and bow with thanks to each other after each serving.

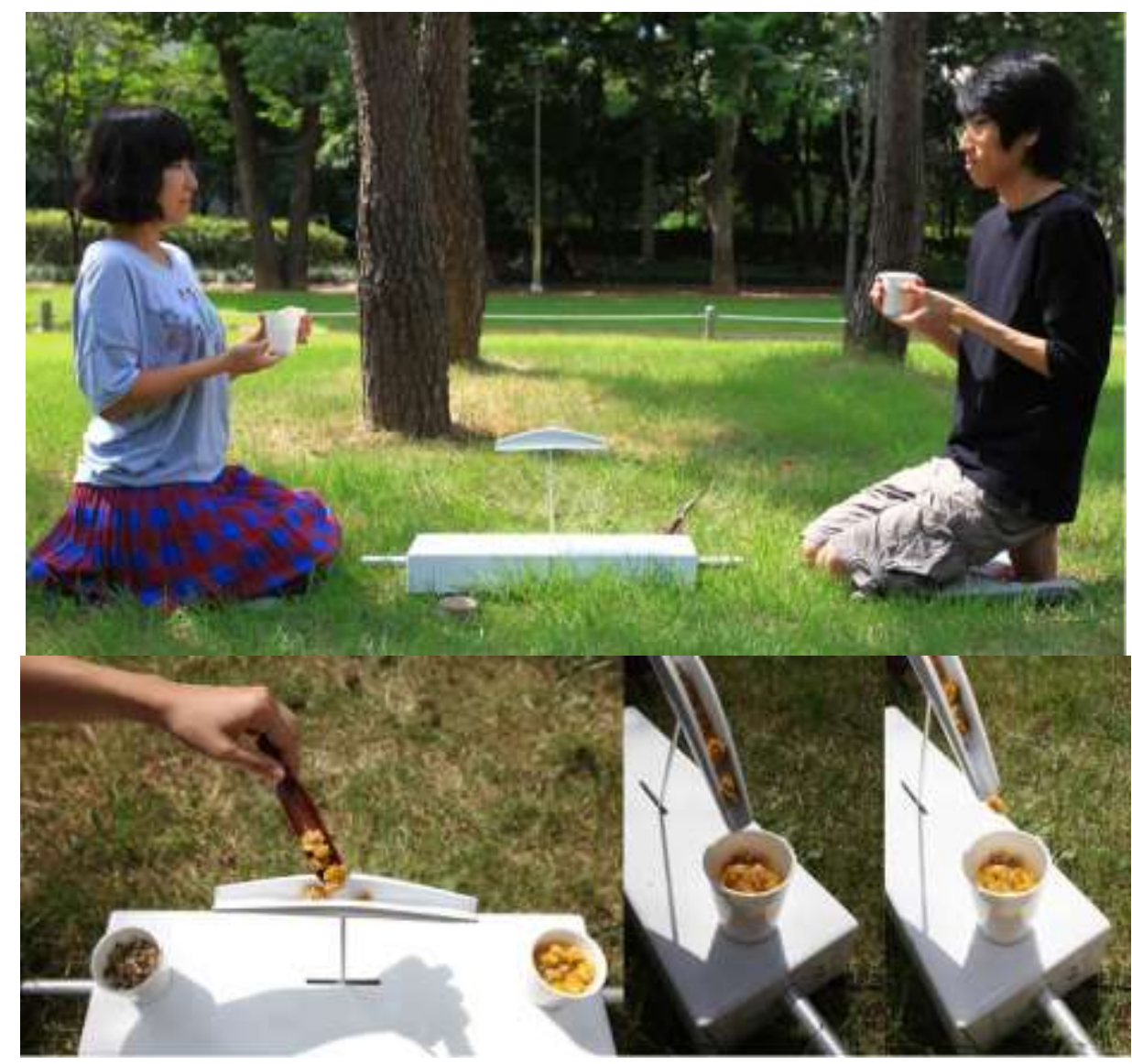

Figure 4. Bow \& Tea 


\subsection{Mechanical Structure}

When designing a custom-made device, the issue is whether the mechanical structure can be easily assembled to increase the quality of a product. In this case, the mechanical parts consist of an acrylic rectangular case, which is $600 \mathrm{~mm}(\mathrm{w}) \times 170 \mathrm{~mm}(\mathrm{l}) \times 70 \mathrm{~mm}$ (h), a metal foundation, two aluminum handles, a thin aluminum pole, and a plastic tea plate.

As the image below shows, four steps are required to put these mechanical parts together:

1) All electronic components - the servo motor, the encoders, and the Arduino processor-are built on a metal foundation. A thin aluminum pole is attached to another metal pole, which is connected to the servo motor across the metal foundation.

2) An acrylic rectangular case is covered with a metal foundation, and an aluminum pole needs to be put into a thin, narrow hole in the center of the case.

3) Two aluminum handles are attached to the rotary encoders at both ends of the foundation, and a tea plate is fastened to the end of the aluminum pole.

4) Two glass cups are placed in the circular-shaped grooves on the top of the whitecover box.

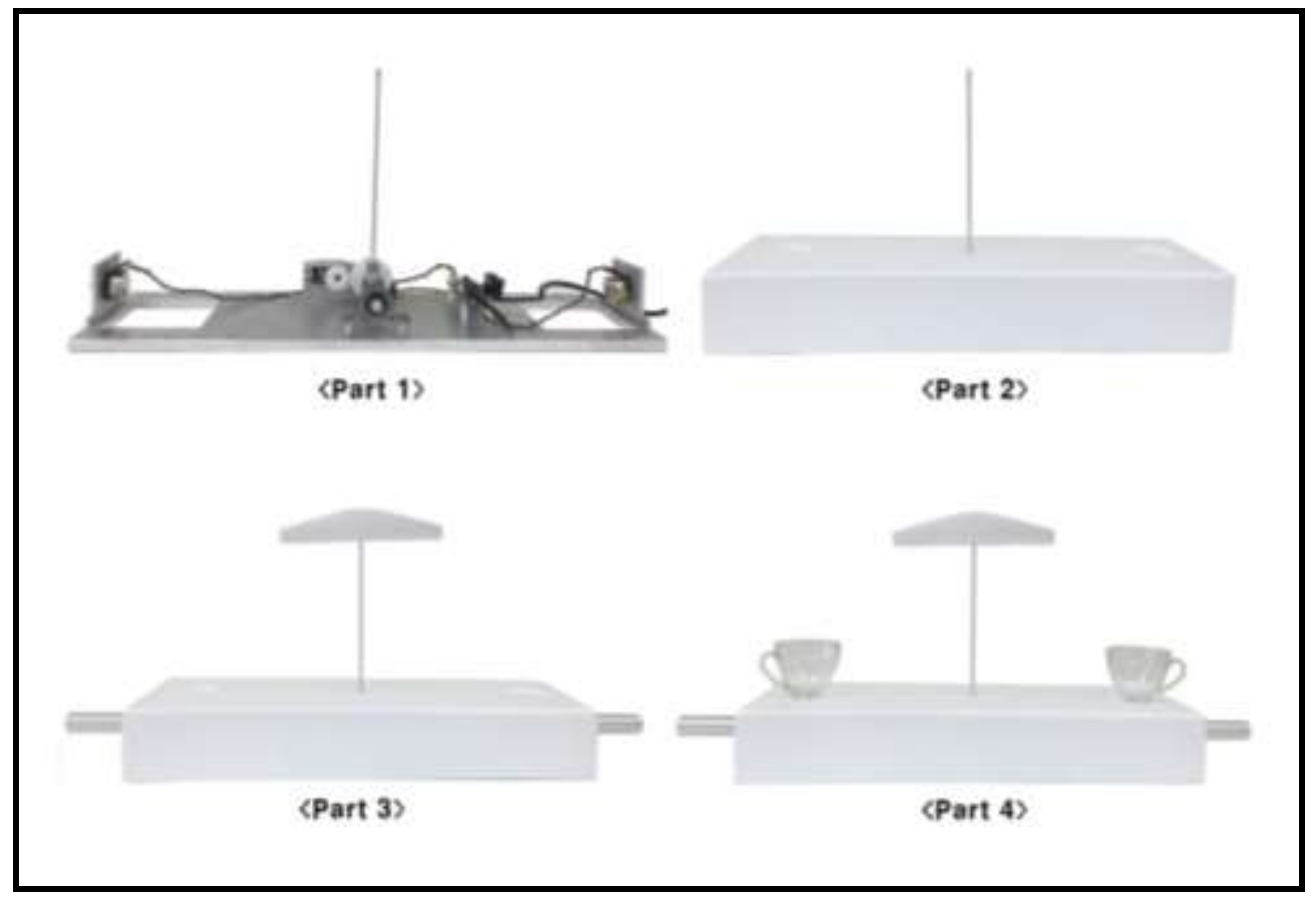

Figure 5. The Process of Assembling Mechanical Parts

\subsection{Design for the User Guide}

On the top of the white case, there are little round grooves that fit into teacups. Users are easily able to focus on these cups when they rotate the handles, so they learn how to drop tea leaves. Another design point for the user's guide is the icon of the tea leaf on both sides of the acrylic rectangular case. This gives information about which direction tea leaves should be dropped in and when to fill a tea plate with leaves. 


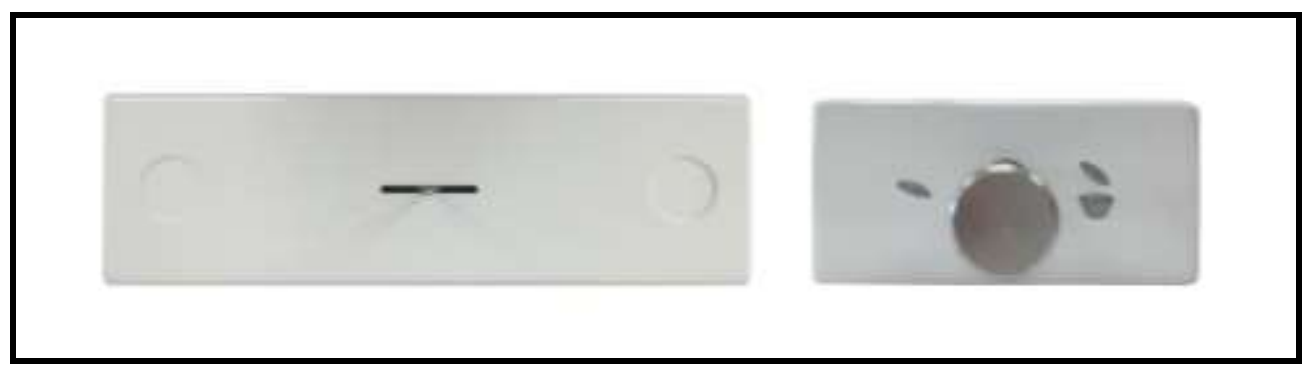

Figure 6. Top and Side View of Bow \& Tea

If the handle is rotated toward the icon of the dropping tea leaves, a tea plate moves down to a teacup on the opposite side. On the other hand, if the handle is rotated toward the icon of the leaf, the tea plate moves up vertically in the middle of the rectangular case. On the top of the white case, a thin, narrow hole for the aluminum pole that supports a tea plate can move up or down and left or right within a 90-degree arc. The tea plate draws the parabola in a bowing-like action as the handle is moved.

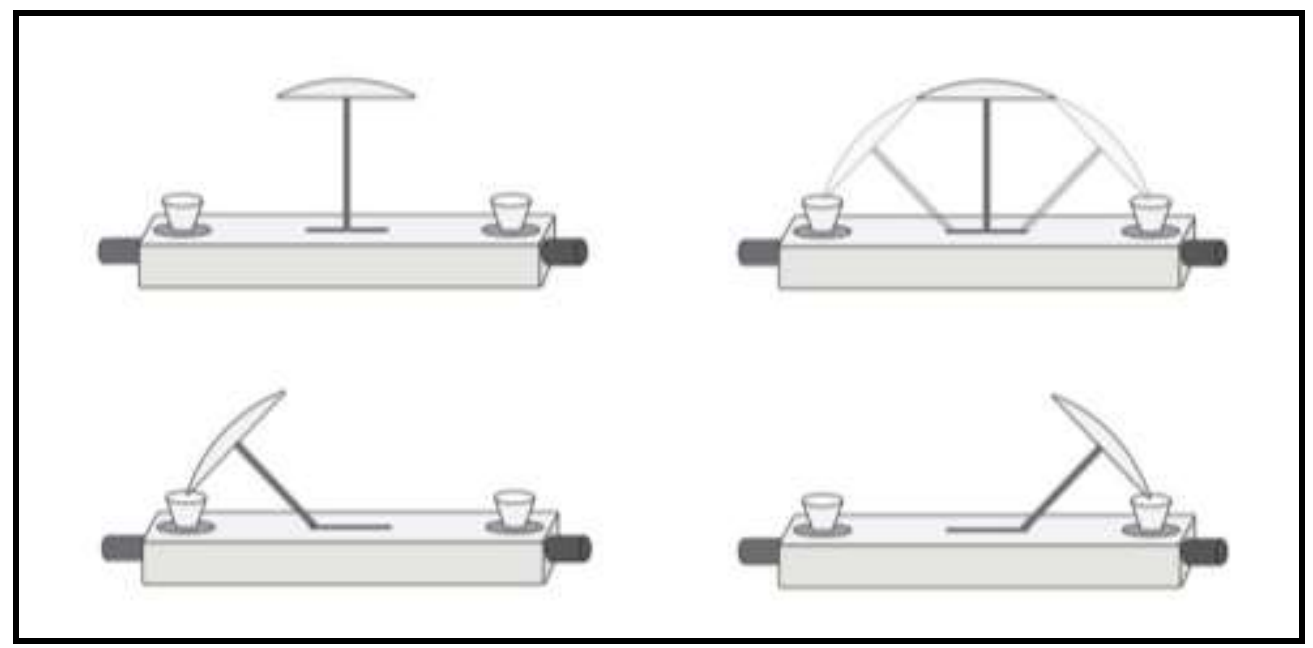

Figure 7. The Mechanical Operation of Bow \& Tea

\subsection{Technical Detail}

The electronic components consist of two rotary encoders, a servo motor, and an Arduino processor. Its technical operation is simple. When a user turns the handles that connect to the rotary encoders, the rotating degrees are sent to the Arduino processor. This processor receives the data and turns it into another form of data, which the servo motor can understand. The transferred data of rotating a certain number of degrees per each servo motor is applied to control the directions and degrees of the servo motor's movement. 


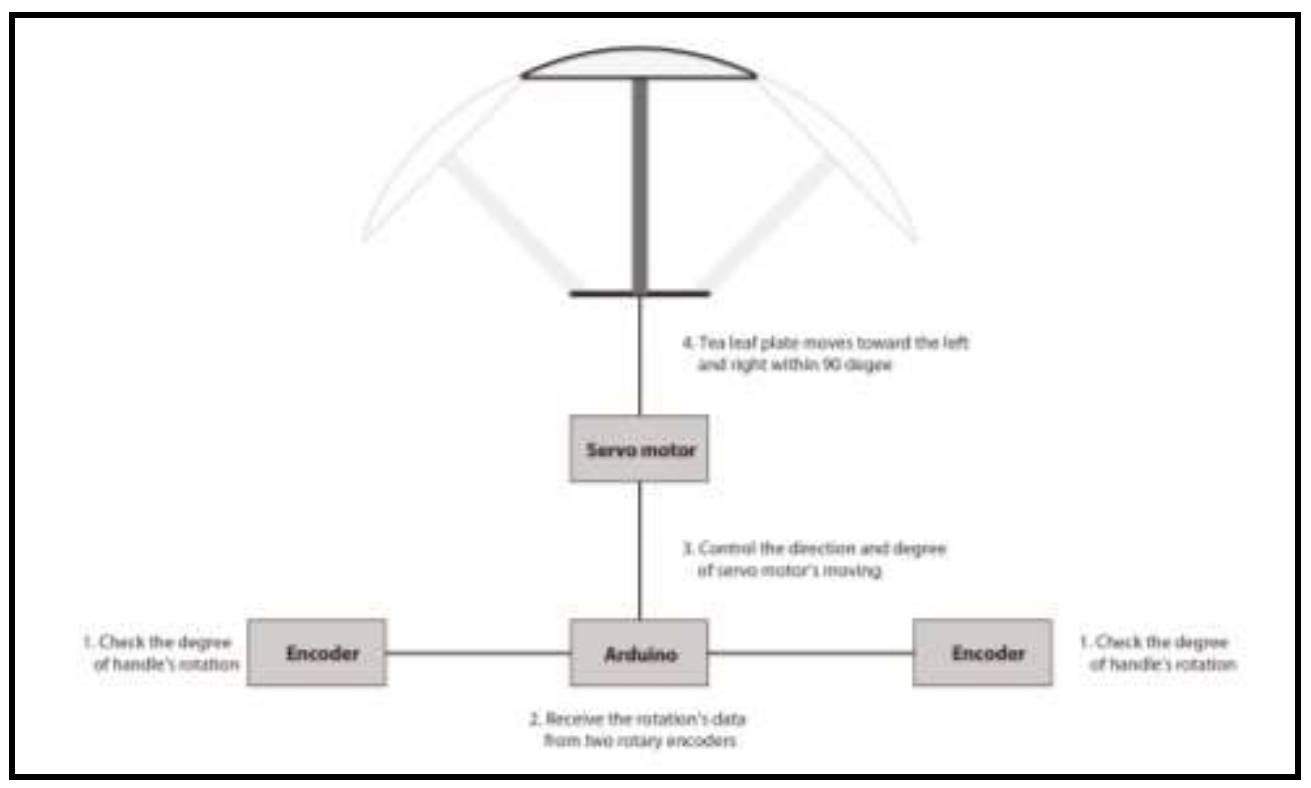

Figure 8. Technical Diagram of Bow \& Tea

In this case study, the servo motor's movement is limited between 0 and 90 degrees, instead of the 180 degree arc that a servo motor generally moves through. Moreover, while the user manipulates one handle, the Arduino processor only takes the data from the first activated encoder, even though another user can manipulate the handle on the opposite side. This is so that two users cannot operate the device at the same time, but they can use it one after the other.

\subsection{Participant' Engagement}

For some, the focus of the tea-making process is a key point when designing the interactivity and playful experience between users and an electronic device, such as Bow $\&$ Tea. The user is required to turn a handle very precisely and control the amount of tea leafs that are dropping into a teacup to maintain good taste. While the handle moves up and down, the tea plate draws the parabola in an arc. It reminds one that bowing is a traditional greeting, as well as a gesture of respect in East Asian culture.

In the Chi-Tek showcase at the Victoria \& Albert Museum and at Watermans Art Centre, it was observed that participants adjusted the tea plate's moving speed to fill the cup with tea leaves for someone whom they had just met. And an unfamiliar server bowed for the maker whenever the tea plate moved. This humorous situation caused them to laugh apart from the functioning of the tea-making machine. Some of the participants also considered Bow \& Tea to be a game. 


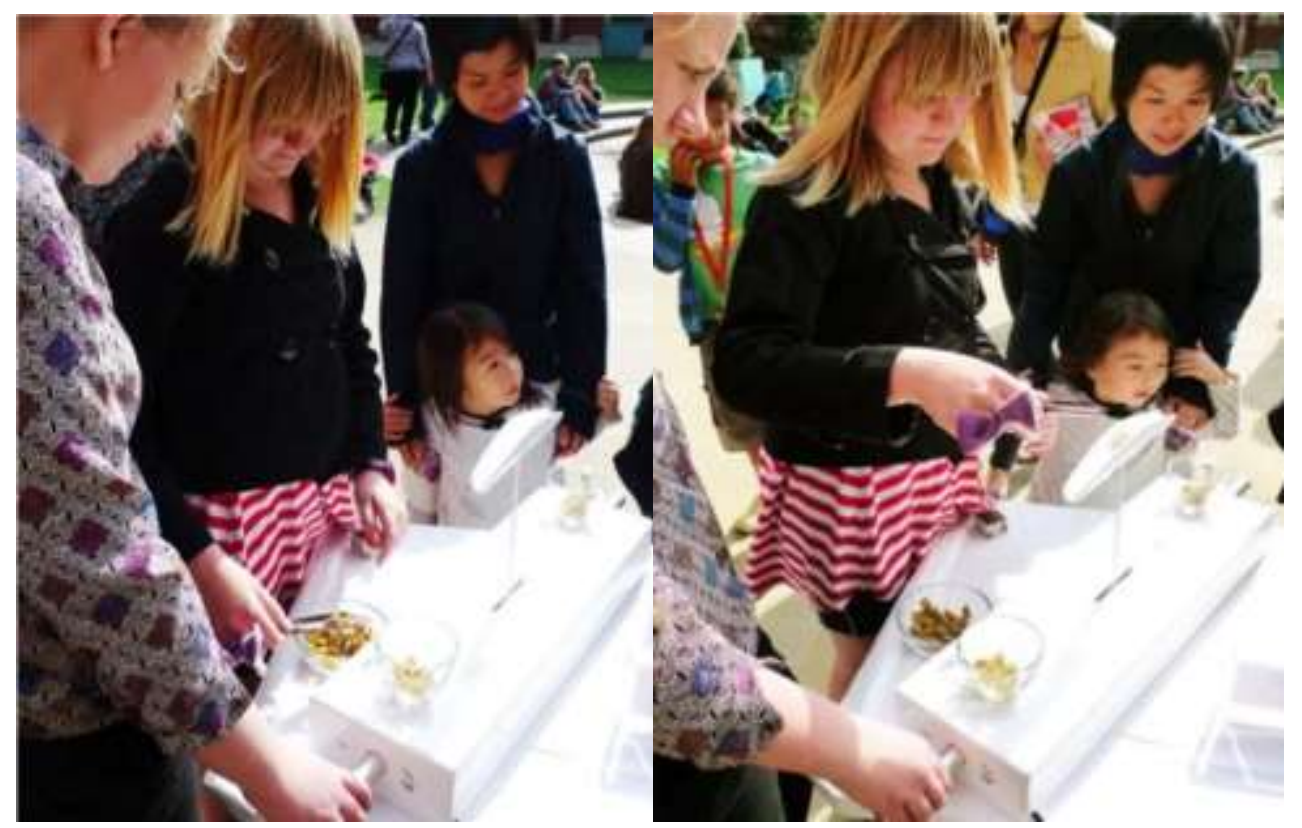

Figure 9. Participations Experiencing Bow \& Tea Outside Victoria \& Albert Museum

\section{Conclusion and Future Work}

In modern society, a technology can be deeply woven into the patterns of everyday life, and we have to consider whether and how we use that technology [15]. Technology has come to not only exist for improving various functions but also as a tool that realizes the creative imagination.

Dunne and Raby's statement describes how we expect art to be shocking and extreme, and that critical design needs to be closer to the everyday, as we know it can be different and that things can change [4], [7]. Machiko Kusahara suggests that Device Art integrating art and technology, as well as design, entertainment, and popular culture, is a new form of media art in Japan. Regardless of how we appreciate the emerging devices in art or design, we now face playful technology-driven devices, which are hard to be merely defined as artwork or design products (e.g., toys and gadgets). Newly appearing devices suggest an approach that life with technology and devices will continue to be suffused with our everyday experiences.

The new devices allow users to enjoy a narrative experience that can be completed through their interactive participation as co-producers. To create these devices, artists use software and hardware instead of traditional art-making media. However, whatever technology is used, it is necessary for it not to be hidden and simple; then users can easily understand the interfaces and functionalities of unleashed devices, or be curious about them.

In this research, the idea of unleashed devices is explored by reviewing related work, as well as exploring the case study of Bow \& Tea. Unleashed devices are open-source projects, produced by artists who explore technologies critically and creatively. Through everyday electronic devices being reconstructed, remixed, and reinvented, they take on a new life as they shift our vision of the use of data and the purpose of technology.

By playing with frontiers, unleashed devices challenge the instrumental conception of technology, as well as art and design. Whatever the reason is for creating interactive, playful devices that allow users to take art out of its original habitat, it is an interesting development. I believe the findings of this case study can significantly assist in future designs of ludic experiences as products for the public. 


\section{Acknowledgments}

This research was supported by the Chung-Ang University Excellent Student Scholarship in 2016. And also the art work presented as a case study in this research was partly funded by Artist Support Program on Human-centered Interaction by Red Pool, a media company in Korea.

\section{References}

[1] H S. H. W. Ossevoort, "From Electric Devices to Electronic Behavior", Transdisciplinary Digital Art. Sound, Vision and the New Screen", Communications in Computer and Information Science, vol. 7, (2008), pp. 392-402.

[2] G. Oliver, "Device Art: A New Approach in Understanding Japanese Contemporary Media Art", the MIT Press, Cambridge, Massachusetts, (2007), pp. 277-309.

[3] M. Kusahara, "Device Art: A New Form of Media Art from a Japanese Perspective", Intelligent agent, vol. 6, no. 2, (2006), pp. 392-402.

[4] A. Dunne, "Electronic Products, Aesthetic Experience and Critical Design", The MIT Press, Cambridge Massachusetts, (2005).

[5] B. Moggridge, "Designing Interactions", The MIT Press, Cambridge Massachusetts, (2006), pp. 237317.

[6] M. Hazzenzahl, "Experience Design: Technology for All the Right Reasons", Morgan and Claypool Publishers, (2010).

[7] A. Dunne and F. Raby, "Speculative Everything: Design, Fiction, and Social Dreaming", The MIT Press, Cambridge, Massachusetts, (2013).

[8] R. Hartson, "Cognitive, physical, sensory, and functional affordances in interaction design", Behaviour \& Information Technology, (2010), pp. 315-338

[9] E. Shin, "Development of an Unleashed Device - Focused on Entertainment Purposes", Proceeding of the 5th International Workshop on Art, Culture, Game, Graphics, Broadcasting and Digital Contents 2016, Jeju, Korea, (2016).

[10] Oxford dictionary, http://www.oxforddictionaries.com/definition/english/device (accessed Aug. 2016)

[11] Tenori-On, http://europe.yamaha.com/en/products/musical-instruments/entertainment/tenori-on/tenorion_series/?mode=series $\% 23 \mathrm{tab}=$ feature\#tab=feature (accessed Aug. 2016)

[12] Bitman, http://www.maywadenki.com/products/goods/bitman/ (accessed Aug. 2016)

[13] Otomatone, http://www.otamatone.com/maywa-denki/ (accessed Aug. 2016)

[14] Tea ceremony, https://en.wikipedia.org/wiki/Tea_ceremony (accessed Aug. 2016)

[15] N. Carr, "Not Addiction; dependency", http://www.roughtype.com/?m=201005 (accessed Aug. 2016)

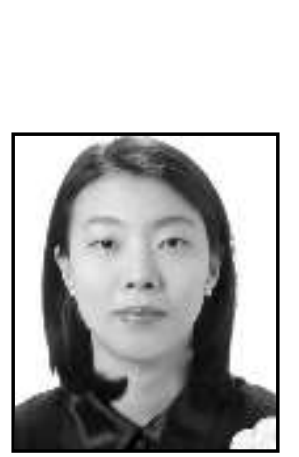

\section{Author}

Eunjoo Shin, Currently, she works an assistant professor of Interactive Design at College of Fine Art and Design, Incheon Catholic University. Her research interests are in the areas of human computer interaction and embodied hardware through creative and social use of technology. 